\title{
A Thermomechanical Transport Approach and Application in Soil-Water System of Polluted Mining Areas considering the Three-Phase Coupling
}

\author{
Xuan Guo, ${ }^{1}$ XiaoXin Zhang, ${ }^{2}$ and MengShu Wang ${ }^{1}$ \\ ${ }^{1}$ School of Civil Engineering, Beijing Jiaotong University, Beijing 100044, China \\ ${ }^{2}$ China Construction Engineering Design Group Corporation Limited, Beijing 100037, China \\ Correspondence should be addressed to Xuan Guo; xguo@bjtu.edu.cn
}

Received 9 January 2016; Accepted 28 February 2016

Academic Editor: Valentin Lychagin

Copyright ( $\odot 2016$ Xuan Guo et al. This is an open access article distributed under the Creative Commons Attribution License, which permits unrestricted use, distribution, and reproduction in any medium, provided the original work is properly cited.

\begin{abstract}
The thermomechanical transport approach includes the process of diffusing or the condition of being diffused, absorption/desorption, swell/shrink, equilibrium/nonequilibrium, and thermomechanical transport of contaminant in three phases of polluted mining soil which are discussed. The thermomechanical transport model of the contaminants transport in polluted soil is established, and its basic equations are given. Based on that, the distribution regularities of the contaminant seepage in water-soil system are discussed in detail and the sensitivities of parameters are analyzed. The study shows that the parameter has important influence on the contamination distribution and transportation in polluted soil-water system. The influence degree is also related to the action of seepage force directly.
\end{abstract}

\section{Introduction}

The thermomechanical transport approach or heat transfer model continues to be a major field of interest to soil engineering and scientific researchers. The main wealth of applications includes a wide variety of components and systems for energy interchanges or mathematic method. (Alonso et al. [1] propose a constitutive model for partially saturated soils; Aziz and Settari [2] give the simulation method of petroleum reservoir; Carslaw and Jagger [3] conduct the heat in solids; Cho et al. [4] present a critical assessment of the use of a surface reaction rate equation to correlate biosorption kinetics; Pedroso [5] gives a solution to transient seepage in unsaturated porous media; Deng et al. [6] put forward the advanced theory and method of fluid flow in porous media; Ibragimov [7] raises the group transformations in mathematical physics; Kanevskaya [8] presents the mathematical modeling of hydrodynamic processes of the development of hydrocarbon deposits; Lykov [9] discussed the theory of heat conduction. Ni et al. [10] proposed a model of groundwater seepage and heat transfer for single-well ground source heat pump systems; Nikolayevskii [11] presented the mechanics of saturated porous media; Lychagin [12] brings the quantizations of differential equations; Volkov et al. [13] put the integrable seepage equations; Rafiezadeh and Ataie-Ashtiani [14] give the seepage analysis in multidomain general anisotropic media by three-dimensional boundary elements; Zhang et al. [15] give the thermohydromechanical-air coupling finite element method and its application to multiphase problems.)

In order to evaluate the movement law of pollutant diffusion under the different hydrodynamic condition more scientifically and predict and prevent diffusing and other involved hazards, the basic work of theory and test needs to be further developed. Due to many unknown macroscopic and microstructure behaviors in mining polluted soil-water system, the thermomechanical transport approach includes the process of diffusing or the condition of being diffused, absorption-desorption, swell/shrink, equilibrium/nonequilibrium, and thermomechanical transport of contaminant in polluted mining soil which are discussed actively.

Porous media of polluted mining area (macromolecular solvent systems) can exhibit absorption-desorption, be diffuse-undiffused, and exhibit swell/shrink resulting in macroscopic behavior which may be significantly different 
from nonpolluted media. It is crucial to understand the constitutive behavior of these materials such as clay or other mining components. Then, the examples of polluted clays are discussed as follows.

\section{Thermomechanical Transport Approach}

Three phases refer to solid, water, and air in soil-water system of polluted mining areas. To simulate the influence of variably saturated flow which is governed by the Darcy equation [16]

$$
Q=\frac{k}{\eta} S \frac{\Delta p}{\Delta l}
$$

while $Q$ is bulk flux; $\Delta p$ is differences in pressure; $\Delta l$ is pathway length; $k$ is infiltration of medium; and $S$ is the area across water.

2.1. Thermohydromechanical-Air Coupling Deformation of Field Equations. The equilibrium equations for polluted geomaterials in a representative elementary volume (REV) are derived [1]: the coupling total stress tensor $F$ and the stress tensors (soil, water, and air) acting on each phase have the following form:

$$
\begin{aligned}
F & =F^{s} \wedge F^{w} \wedge F^{a}, \\
F^{s} & =F^{\prime}+(1-n) p^{F} I, \\
F^{w} & =n S_{r} p^{w} I, \\
F^{a} & =n\left(1-S_{r}\right) p^{a} I,
\end{aligned}
$$

where $I$ is the identity tensor, $F^{\prime}$ is the effective stress tensor,

$$
\begin{aligned}
F & =F^{\prime}+p^{F} I, \\
\dot{F} & =\dot{F}^{\prime}+\dot{p}^{F} I, \\
p^{F} & =S_{r} p^{w}+\left(1-S_{r}\right) p^{a},
\end{aligned}
$$

the densities of soil, liquid, and air, respectively, are $\rho^{s}, \rho^{w}$, and $\rho^{a}, n$ is the porosity of the soil, and $S_{r}$ is the degree of saturation. The appearance densities of the solid phase, the liquid phase, and the air phase are defined as $\bar{\rho}^{s}, \bar{\rho}^{w}$, and $\bar{\rho}^{a}$,

$$
\rho=(1-n) \rho^{s}+n\left[S_{r} \rho^{w}+\left(1-S_{r}\right) \rho^{a}\right] .
$$

The mass conservation law for the solid, liquid, and air phases in the local form can be written as

$$
\begin{gathered}
\frac{\partial \bar{\rho}^{s}}{\partial t}+\operatorname{div}\left(\bar{\rho}^{s} v^{s}\right)=0 \\
\frac{\partial \bar{\rho}^{w}}{\partial t}+\operatorname{div}\left(\bar{\rho}^{w} v^{w}\right)=0 \\
\frac{\partial \bar{\rho}^{a}}{\partial t}+\operatorname{div}\left(\bar{\rho}^{a} v^{a}\right)=0 .
\end{gathered}
$$

The equilibrium equation of soil-water-air three-phase field theory can be given as

$$
\operatorname{div} \mathbf{T}+\rho \mathbf{b}=0 .
$$

The thermomechanical transport approach includes the process of diffusing or the condition of being diffused, absorption/desorption, swell/shrink, equilibrium/nonequilibrium, and thermomechanical transport of contaminant in three phases of polluted mining soil which can be compactly involved in the above equations.

2.2. Equation of Energy Conservation and Flow Rules. Conform the process of being diffuse/undiffused, absorption/ desorption, swell/shrink, and equilibrium/nonequilibrium as the uniform dual uncoupling efforts. From the first/second law of thermodynamics, the unit work increment is

$$
d W=d \Phi+d E=p^{\prime} d \varepsilon_{V}+q d \varepsilon_{S} .
$$

The basic requirement of the proposed model within the thermodynamical framework satisfies the basic laws of physics.

The energy increment of the unit is

$$
d E=\frac{\partial E}{\partial \varepsilon_{V}} d \varepsilon_{V}+\frac{\partial E}{\partial \varepsilon_{S}} d \varepsilon_{S}+\sum_{\substack{i=D, R \\ j=V, S}} \frac{\partial E}{\partial \varepsilon_{j}^{i}} d \varepsilon_{j}^{i} .
$$

Dissipation mechanism of the analysis unit is supposed to associate with plastic deformation only; dissipation potential increment can be given by Euler theorem as

$$
d \Phi=\sum_{\substack{i=D, R \\ j=V, S}} \frac{\partial(d \Phi)}{\partial \varepsilon_{j}^{i}} d \varepsilon_{j}^{i},
$$

where the index of $D, R$ and $V, S$ refers to the mechanics of the process of being diffuse/undiffused, absorption/desorption, swell/shrink, and equilibrium/nonequilibrium, respectively.

Then, the thermodynamical generalized stress can be defined:

$$
\begin{aligned}
p^{\prime} & =\frac{\partial E}{\partial \varepsilon_{V}} ; \\
q & =\frac{\partial E}{\partial \varepsilon_{S}} ; \\
-\frac{\partial E}{\partial \varepsilon_{j}^{i}} & =\frac{\partial(d \Phi)}{\partial \varepsilon_{j}^{i}}=: \chi_{j}^{i}
\end{aligned}
$$

\subsection{Transformation between Generalized Stress Space and} True Stress Space. As the transformation relationship between generalized stress space and true stress space is valuable for engineering analysis, the transformation from the generalized dissipative space to the true stress space is proposed. The shift or back stresses are used to account for the effect of the translating yield without changing the shape.

From the basic energy relation of plastic work increment relationship, the shift (or back) stress for transport approach can be termed as

$$
\alpha_{j}^{i}=: \frac{\partial E^{P}}{\partial \varepsilon_{j}^{i}} ; \quad(i=D, R ; j=V, S) .
$$


The transformation relations between generalized stress space and true stress space can be given as

$$
\begin{aligned}
& p^{\prime}=\chi_{V}^{i}+\alpha_{V}^{i} ; \\
& q=\chi_{S}^{i}+\alpha_{S}^{i} ; \quad(i=D, R) .
\end{aligned}
$$

The physical phenomena of being diffuse/undiffused, absorption/desorption, swell/shrink, and equilibrium/nonequilibrium can be simulated by the dual effects. Accordingly the yield surfaces of the dual efforts satisfy the associated plastic flow rule and follow the degenerate special case of Legendre transformation.

The relations in generalized dissipation space are given:

$$
\mu^{i} F^{i}=\sum_{j=V, S} \chi_{j}^{i} d \varepsilon_{j}^{i}-d \Phi^{i}=0 ; \quad(i=D, R) .
$$

The corresponding flow rules are

$$
d \varepsilon_{j}^{i}=\mu^{i} \frac{\partial F^{i}}{\partial \chi_{j}^{i}} ; \quad(i=D, R ; j=V, S) .
$$

The dual efforts of being diffuse/undiffused, absorption/ desorption, swell/shrink, and equilibrium/nonequilibrium are derived by transformation of shift stress in true stress space as

$$
\mu^{i} f^{i}=\left(p^{\prime}-\alpha_{V}^{i}\right) d \varepsilon_{V}^{i}+\left(q-\alpha_{S}^{i}\right) d \varepsilon_{S}^{i}-d \Phi^{i}
$$

Corresponding flow rules in true stress space can be derived as

$$
\begin{aligned}
d \varepsilon_{V}^{i}=\mu^{i} \frac{\partial f^{i}}{\partial p^{\prime}} ; & \\
d \varepsilon_{S}^{i}=\mu^{i} \frac{\partial f^{i}}{\partial q} ; & \\
& (i=D, R) .
\end{aligned}
$$

\section{A Seepage Model in Unsaturated Porous Soil-Water Media}

Uncertainty of groundwater is one of the factors that most influence polluted mining area. This is also one of the most difficult to characterize. In most geological environments groundwater is largely confined in fractures and shear zones, and its impact on polluted mining area is usually related to the intersection of faults by the mining table. Although fractures and shear zones do not have a large volume of stored water, they serve as channels and favored flow paths collecting and concentrating water from the rock-soil mass and then further influence the soil-water polluted characterization and classification. Identification of fractured zones and shear zones both from the structural and from hydraulic considerations for the polluted characterization and classification is important.
Measuring, describing, or defining the parameters or properties related to seepage is important. Uncertainties of ground conditions are derived from spatially stochastic distribution and transformation, and it caused the random variation of the seepage coefficients. The geological seepage model is developed and the according characterization is processed. The quantified characterization for a mining table would consist of described discontinuity rock characteristics, geometric distribution, and orientation of these joints. The hydrogeology characterizations of groundwater help to understand the seepage behavior.

It is known that many uncertainty factors may affect the parameter estimation of soil-water polluted seepage model or force. The flow of fluids within the porous medium is expressed in terms of a thermomechanical framework. For the seepage modeling, the solid matrix is considered to be perfectly rigid and the seepage velocity of liquid $v_{l}$ is introduced. $v_{l}$ allow for the definition of a viscous interaction. With basis on the dissipation inequality, the following model form that can recover seepage model for steady saturated flow is assumed:

$$
\hat{p}_{l}=\frac{\partial n_{l}}{\partial x} p_{l}-\frac{n_{l}^{2} g \rho^{l}}{k_{l}^{r}\left(\underline{k}_{l}^{\text {unsat }}\right)} \cdot v_{l},
$$

where the interaction term in the balance of linear momentum is $\hat{p}_{\cdot l}, g$ is the gravity acceleration, $k_{l}^{t}$ is a multiplier that depends on the liquid saturation, and $k^{\text {unsat }}$ is a tensor analogous to conductivity parameter. The permeability coefficient is expressed in tensor form in anisotropic media. The greater the permeability coefficient is, the stronger the rock and contaminant permeability ability is.

The hydraulic conductivity tensor function on unsaturated polluted soil-water system can be simplified following common modeling approach. A multiplier $0 \leq k_{l}^{t}\left(s_{l}\right) \leq 1$ is defined for the dependence of hydraulic conductivity on unsaturation soil-water system. Then, the liquid conductivity model function and derivative are

$$
k_{l}^{t}\left(s_{l}\right)=\lambda_{0 l} s_{l}-\frac{1}{\beta_{l}} \log \left(c_{3}+c_{2} e^{c_{1} s_{l}}\right)
$$

with

$$
\begin{aligned}
& c_{1}=\beta_{l}\left(\lambda_{0 l}-\lambda_{1 l}\right) ; \\
& c_{2}=e^{-\beta_{l} \alpha_{l}} ; \\
& c_{3}=e^{\beta_{l}\left(\lambda_{0 l}-1\right)}-c_{2} e^{c_{1}},
\end{aligned}
$$

while $\lambda$ is the slope of the seepage gradient in $k_{l}^{t}-s_{l}$ plane and $\beta_{l}$ parameter is given constant number. In the case of the one-dimensional isothermal model of a rectilinearparallel seepage flow, the permeability coefficient of coarse gravel is more than $10 \mathrm{~m}$ per day/night; the permeability of the stone-sand-soil mixture loam varies from 1 to $0.01 \mathrm{~m}$ per day/night; the permeability coefficient of impermeable clay is less than $0.001 \mathrm{~m}$ per day/night. The soil permeability 


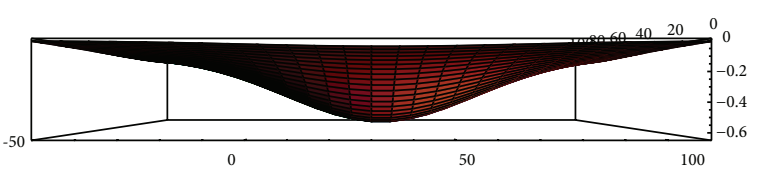

(Unit: $\mathrm{m}$ )

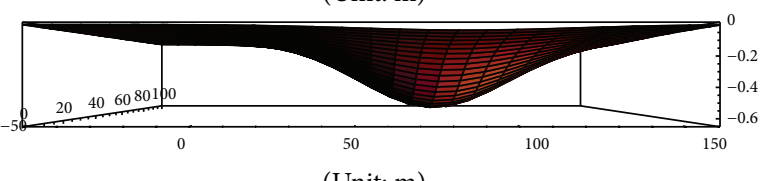

(Unit: m)

FIGURE 1: Section of the three-dimensional movement analysis of the contamination seepage.

coefficients depend on the process of diffusing or the condition of being diffused, absorption/desorption, swell/shrink, equilibrium/nonequilibrium, and thermomechanical transport of contaminant in three phases of polluted mining soil accordingly.

\section{Three-Dimensional Transport Simulation and Control}

Construction of the mining table that proceeded needs to control the developing seepage and soil-water pressure during the whole polluted mining area process. It is necessary to control the seepage rate to accommodate the ground adaptability characterization. The effective seepage control relies on the statistical parameter estimation. For example, the problem found in a China river cross mining table was related to the state of high soil-water seepage force and stress and effective control of seepage force can well balance to minimize construct risk in a statistical parameter scope.

A simple 3D-simulation example of the seepage model in unsaturated porous soil-water media is given as follows. In the area of $100 \mathrm{~m}$ length, $100 \mathrm{~m}$ width, and $0.6 \mathrm{~m}$ depth, the contamination migration rule of a soil-water system is shown in Figures 1 and 2. The cases of contamination threedimensional seepage movement of Figures 1 and 2 can show the common effect of mitigation application in most polluted mining area projects. Inside, the three-dimensional seepage movement of polluted wave transport approach can be characterized in Figure 1. The planform for three-dimensional movement analysis of the contaminant seepage is presented in Figure 2. This case can degenerate to the single factor and single level analysis. Seepage migration can be realized clearly and it is known that risk can be controlled by restricting the scope and rate of seepage force and hydrodynamic condition.

The simulation test can study the movement law of pollutant diffusion under the different seepage coefficient and hydrodynamic condition in soil-water system. Pollutant extends its incidence by diffusing after dissolving and being carried by soil-water flow. The transport of pollutants seepage could be surprisingly wide and rapid. By this method, predicting the diffusion of pollutant, managing and controlling soil-water quality can be done.
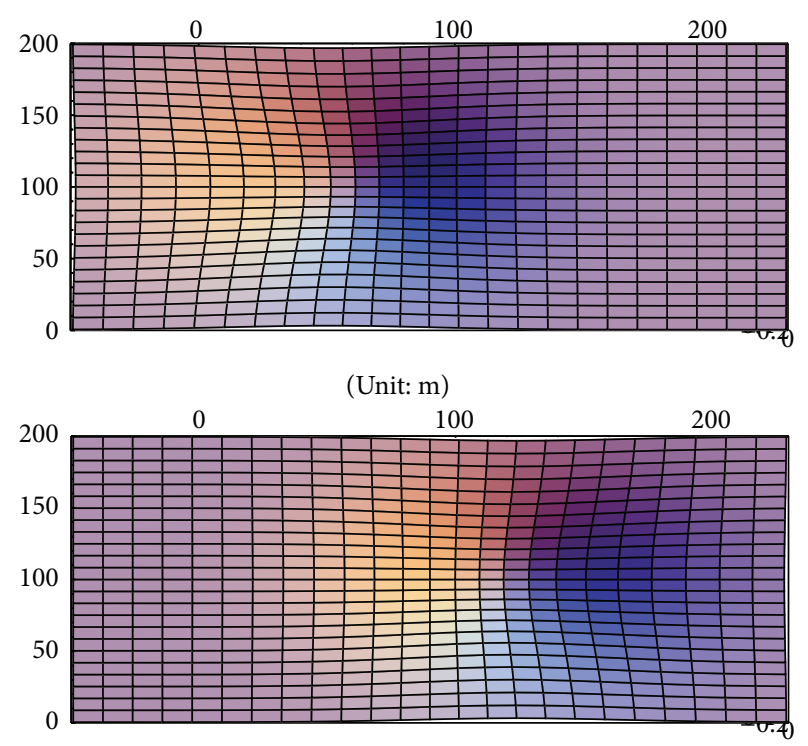

(Unit: $\mathrm{m}$ )

FIgURE 2: Platforms of three-dimensional movement analysis of the contamination seepage.

In the practice, because it takes very long time to get balanced temperature, herein a fixed characteristic temperature is proposed to weaken its influence in this paper.

It can be seen from Figures 1 and 2 that the threedimensional seepage disperses fast in the soil-water system. We can think that pollutant fast extends its incidence by diffusing after dissolving and being carried by flowing. Then, control dispersion of multiphase contaminants in soilwater system to protect occupants in polluted mining areas and environment is important. Grouting, increase of solid components, or green antiseepage plugging agent is the good way to consider in the practice engineering project.

So, the case study of pollutant dispersion and distribution is reported.

\section{Conclusions}

The use of a thermomechanical transport approach and its application in soil-water system of polluted mining areas considering three-phase coupling models can lead to the deeper understanding of the soil-water behavior during the polluted mining area process.

The thermomechanical transport approach includes the process of diffusing or the condition of being diffused, absorption/desorption, swell/shrink, equilibrium/nonequilibrium, and thermomechanical transport of contaminant in three phases of polluted mining soil which are simply discussed. The thermomechanical transport model of the contaminants transport in polluted soil is directly related to the thermomechanical seepage force, and its finite difference equation is given. Based on it, the distribution regularities of the contaminant in soil-water system are discussed in detail, and the sensitivities of parameters are analyzed. The study shows that the seepage parameters have important influence 
on the function distribution, and the larger $k$ is, the more determined the influence is.

In summary, a 3D thermomechanical transport approach and application in soil-water system of polluted mining areas considering the three-phase coupling are investigated. Rooted early form the Darcy formula and its modified transformation, the random and effective thermomechanical transport approach and its application in soil-water system of polluted mining areas considering three-phase coupling method in the time domain should be explored detailedly in the future.

\section{Competing Interests}

The authors declare that they have no competing interests.

\section{Acknowledgments}

This work was supported by "the National Natural Science Foundation of China (no. 51378051), the Fok Ying Tung Education Foundation (no. 122009), and the Fundamental Research Funds for the Central Universities no. 2011JBZ008."

\section{References}

[1] E. E. Alonso, A. Gens, and A. Josa, "A constitutive model for partially saturated soils," Geotechnique, vol. 40, no. 3, pp. 405430, 1990.

[2] K. Aziz and A. Settari, Petroleum Reservoir Simulation, Applied Science Publishers, London, UK, 1979.

[3] H. S. Carslaw and J. C. Jagger, Conduction Heat in Solids, Clarendon Press, Oxford, UK, 1959.

[4] D. H. Cho, K. H. Chu, and E. Y. Kim, "A critical assessment of the use of a surface reaction rate equation to correlate biosorption kinetics," International Journal of Environmental Science and Technology, vol. 12, no. 6, pp. 2025-2034, 2015.

[5] D. M. Pedroso, "A solution to transient seepage in unsaturated porous media," Computer Methods in Applied Mechanics and Engineering, vol. 285, pp. 791-816, 2015.

[6] Y. E. Deng, X. Q. Liu, R. Q. Huang et al., Advanced Theory and Method of Fluid Flow in Porous Media, Science Press, Beijing, China, 2004 (Chinese).

[7] N. Kh. Ibragimov, Group Transformations in Mathematical Physics, Nauka, Moscow, Russia, 1983.

[8] R. D. Kanevskaya, Mathematical Modeling of Hydrodynamic Processes of the Development of Hydrocarbon Deposits, IKI, Moscow, Russia, 2003.

[9] A. V. Lykov, Theory of Heat Conduction, Vyssh Shkola, Moscow, Russia, 1967.

[10] L. Ni, H. Li, Y. Jiang, Y. Yao, and Z. Ma, "A model of groundwater seepage and heat transfer for single-well ground source heat pump systems," Applied Thermal Engineering, vol. 31, no. 14-15, pp. 2622-2630, 2011

[11] V. N. Nikolayevskii, The Mechanics of Saturated Porous Media, Nedra, Moscow, Russia, 1970.

[12] V. Lychagin, "Quantizations of differential equations," Nonlinear Analysis: Theory, Methods \& Applications, vol. 47, no. 4, pp. 2621-2632, 2001.
[13] V. G. Volkov, L. R. Galiakberova, and I. S. Zheltova, "Integrable filtration equations," Journal of Applied Mathematics and Mechanics, vol. 76, no. 2, pp. 256-264, 2012.

[14] K. Rafiezadeh and B. Ataie-Ashtiani, "Seepage analysis in multi-domain general anisotropic media by three-dimensional boundary elements," Engineering Analysis with Boundary Elements, vol. 37, no. 3, pp. 527-541, 2013.

[15] F. Zhang, Y. Xiong, S. Zhang, and B. Ye, "Thermo-hydromechanical-air coupling finite element method and its application to multi-phase problems," Journal of Rock Mechanics and Geotechnical Engineering, vol. 6, no. 2, pp. 77-98, 2014.

[16] G. O. Brown, "Henry Darcy and the making of a law," Water Resources Research, vol. 38, no. 7, pp. 11-1-11-12, 2002. 


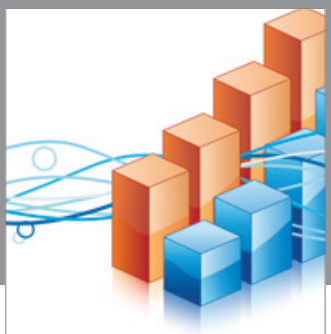

Advances in

Operations Research

vatem alat4

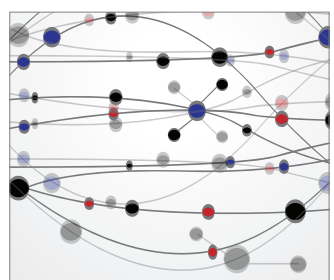

\section{The Scientific} World Journal
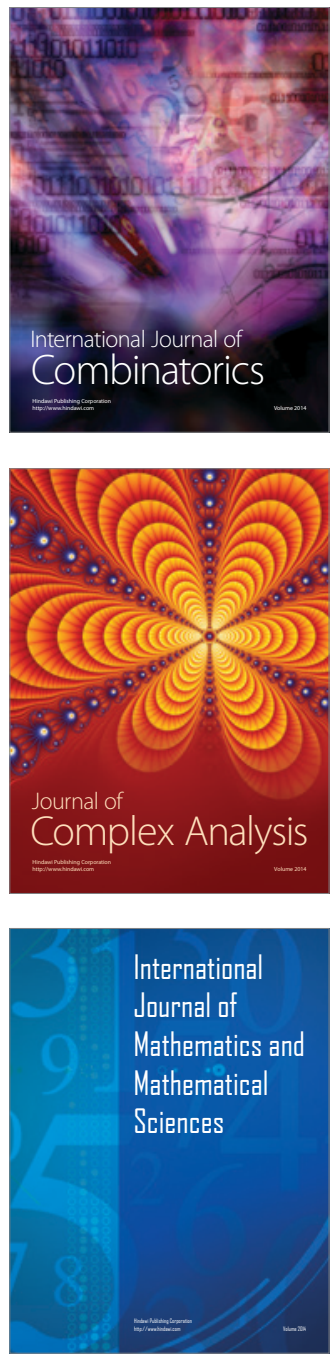
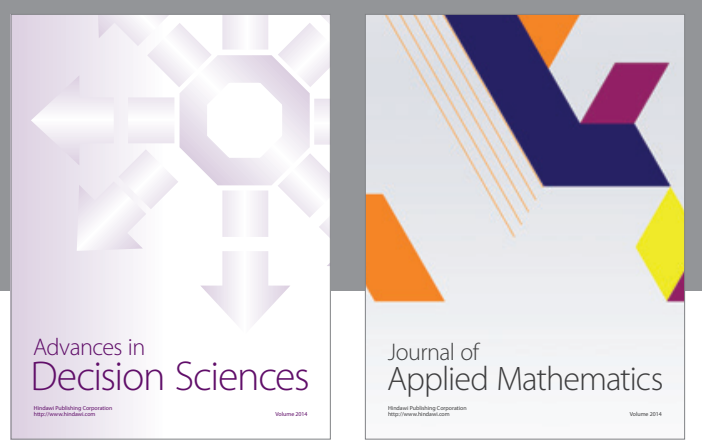

Algebra

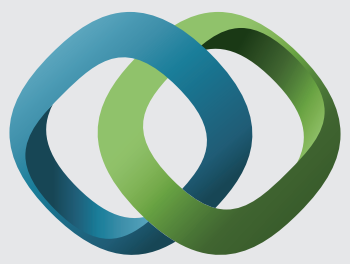

\section{Hindawi}

Submit your manuscripts at

http://www.hindawi.com
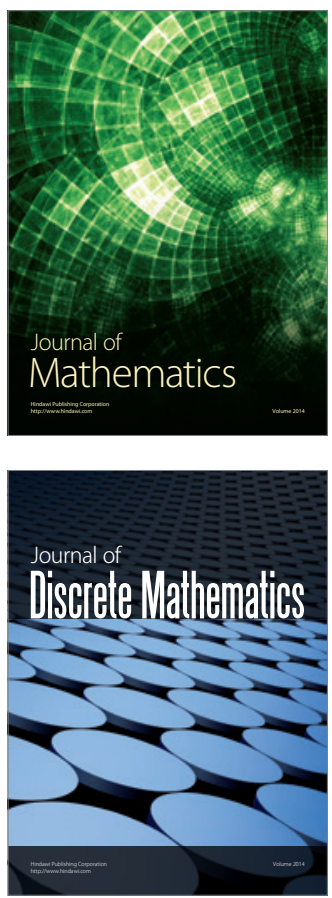

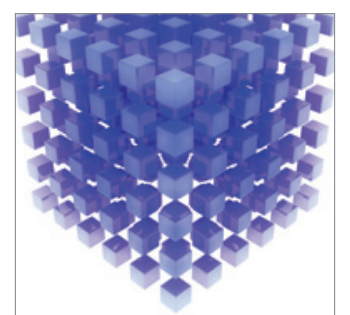

Mathematical Problems in Engineering
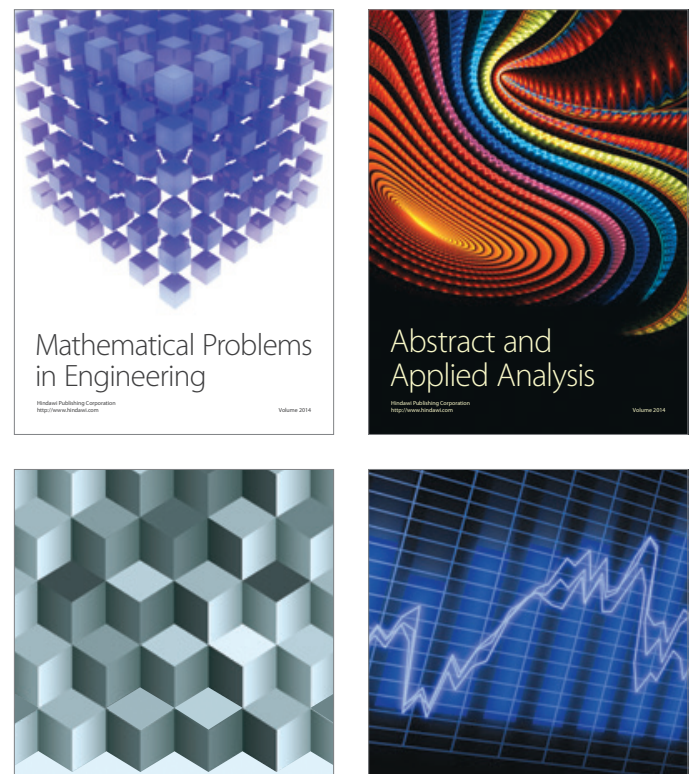

Journal of

Function Spaces

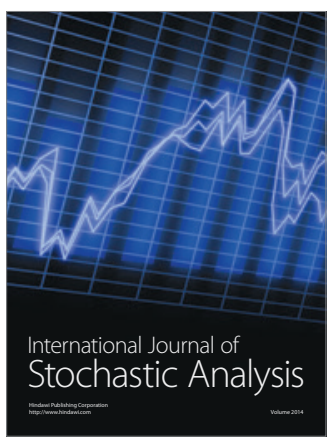

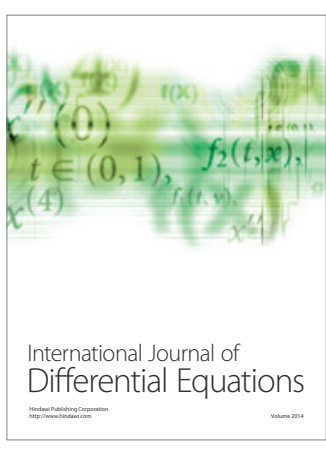
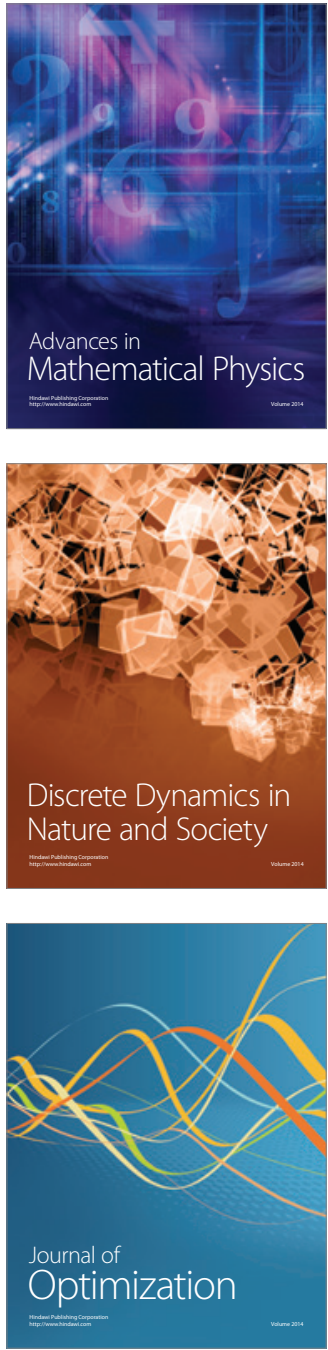